\title{
Association Between Cannabis Use and Sexual Risk Behavior Among Young Heterosexual Adults
}

\author{
Jeannette Brodbeck, ${ }^{1,2}$ Monika Matter, ${ }^{1}$ and Franz Moggi ${ }^{1}$
}

\begin{abstract}
Published online: May 12, 2006
To study the association between cannabis use and frequent sexual risk behavior, we tested the hypothesis of a situational influence of cannabis use in sexual encounters using a combination of global association study and event-level analysis and examined possible mediator variables, including the personality trait of hedonism/risk preference, psychosocial stress, and HIV-related beliefs, using mediation models. The results of a computer-assisted telephone interview of a random sample of 2790 heterosexual men and women aged 16-24 years showed that risky sexual behavior was more frequent in cannabis-using men and women than in nonusing persons. The results did not support a situational effect of cannabis intoxication on sexual risk behavior. The more frequent sexual risk behavior among cannabis users was mediated by decreased intentions to use HIV protection, by lower HIV-self-efficacy, and higher risk preference/hedonism. Only among women psychosocial stress was a partial mediator. The findings show that HIV prevention programs for cannabis-using young adults should emphasize the role of person variables instead of situation variables.
\end{abstract}

KEY WORDS: cannabis; sexual risk; HIV; young adults; mediation.

\section{INTRODUCTION}

Cannabis users show more sexual risk behavior than non-users and are at higher risk for HIV infection and sexually transmitted diseases (Bon, Hittner, and Lawandales, 2001; Castilla, Barrio, Belza, and de la Fuente, 1999; Poulin and Graham, 2001). Cannabis use is also related to early initiation of sexual activity (Staton et al., 1999) and a greater number of sexual partners (Poulin and Graham, 2001; Wingood and DiClemente, 1998). Different models of the association between substance use and unprotected sex (e.g., Stall, McKusick, Wiley, Coates, and Ostrow, 1986) can be applied to explain the association of cannabis use and sexual risk behavior. Situational effects of cannabis intoxication, such as impairment of information processing (Kelleher, Stough, Sergejew, and Rolfe, 2004), relaxation or eu-

\footnotetext{
${ }^{1}$ University Hospital for Clinical Psychiatry, Berne, Switzerland.

${ }^{2}$ Correspondence should be directed to Jeannette Brodbeck, University Hospital of Clinical Psychiatry Berne, Bolligenstrasse 111, 3000 Berne 60, Switzerland; e-mail: brodbeck@puk.unibe.ch.
}

phoric mood (Green, Kavanagh, and Young, 2003), and self-reported aphrodisiac effects (Kleiber and Soellner, 1998), could reduce behavioral control and increase the risk of unprotected intercourse. Alternatively, an underlying risk disposition could induce both cannabis use and sexual risk. Stall et al. (1986) discuss personality factors, a common social context, lifestyle, or a combination of these factors as possible "third variables" that influence risk disposition.

Whereas, some studies find global associations between substance use and sexual risk, event-level analyses generally do not support the hypotheses of a situational influence of drinking or drug use on sexual risk behavior (e.g., Gillmore et al., 2002; Leigh, 1999; Weinhardt and Carey, 2000). To date, the effect of cannabis use on sexual risk behavior among young adults has not been analyzed in a systematic assessment of situational effects and underlying risk disposition.

This study examined two questions regarding the effect of cannabis on sexual risk. First, to what extent are drug effects on sexual risk behavior due to the immediate, situational use of the substance 
versus stable features of the person that induce both cannabis use and risk. Second, if both cannabis use and sexual risk reflect an underlying risk disposition, what specific person variables underlie that disposition?

The theories of Bengel (1996) and Catania, Kegeles, and Coates (1990) would predict a mainly situational effect of cannabis intoxication on sexual risk behavior. Bengel (1996) modified Roger's revised Protection Motivation Theory (Rogers, 1983) for the domain of HIV to model HIV protection intentions and HIV protective behavior. An important adaptation is the addition of intervening variables, such as partner interaction or drug intoxication, between the intention to use HIV protection and HIV protective behavior. The AIDS Risk Reduction Model (ARRM) (Catania et al., 1990) also considers substance use as a contextual factor in the post-intentional enactment stage, which might undermine condom use. If sexual risk behavior is mainly due to a situational effect of cannabis use, interventions should focus on situational variables, skills of condom application or condom negotiation under the influence of cannabis, or avoiding cannabis use in the context of possible sexual encounters.

Other theories emphasize an underlying risk disposition and "third variables" like personality factors, psychosocial stress, or a common social context, including peer group norms toward health behavior that could lead to a higher risk of cannabis use and sexual risk behavior (e.g., Stall et al., 1986). Jessor and Jessor's Problem Behavior Theory (Jessor and Jessor, 1977), on which many studies on risk behavior have based, assumes that different risk behaviors correlate highly and share common underlying conditions of personality, environment, and social behavior that increase proneness for risk behavior (Jessor, 2001). The relationship between substance use and unprotected sex may reflect an underlying risk disposition based on personality factors such as hedonism or risk orientation. Psychosocial stress in important life contexts, such as family, friendship, or work, could lead to substance use or risky sex as coping behavior or as an expression of a general indifference towards health. Also social cognitive models of sexual risk behavior like the Theory of Planned Behavior (Ajzen, 1991) and the Health Belief Model (see Becker, 1974) emphasize person variables, such as intention to use condoms, selfefficacy, and subjective norms, which, as proximal behavior variables, have a high predictive value for explaining sexual risk behavior (Sheeran, Abraham, and Orbell, 1999). If sexual risk behavior among cannabis users is due mainly to an underlying risk disposition, interventions should focus on person variables that influence this risk disposition.

Consistent with the Problem Behavior Theory and social cognitive models of sexual risk behavior we hypothesized that the personality trait of higher risk preference and hedonism, psychosocial stress, intention to use HIV protection and HIV selfefficacy, would be key mediators of the linkage between cannabis use and sexual risk behavior. The importance of the peer group for substance use among adolescents (Hawkins, Catalano, and Miller, 1992) is well documented as well as the influence of peer or partner norms on condom use (Sheeran and Taylor, 1999) and the intention to use condoms (Sheeran and Taylor, 1999) and not the focus of the present study.

To provide a better understanding of the relationship between cannabis use and sexual risk behavior, the present study combined two research strategies, the event-level and global association methods. If cannabis use leads to sexual risk due to its immediate effects in the sexual situation, we would expect that individuals who used cannabis prior to the most recent sexual intercourse event would be more likely to show risk than individuals who did not use cannabis and that the prevalence of using cannabis is higher in the last unprotected than in the last protected intercourse event. Significant effects in global association data, but limited effects in more event-level data would support a more "person" based perspective. Having both event-level and global data allowed us to test these two perspectives.

\section{METHODS}

\section{Participants}

A random sample of 16-24-year-old urban Swiss men and women was selected based on the official registers of the Residents' Administration Offices of the Swiss cities of Basel, Bern, and Zurich. Data were collected by computer-assisted telephone interview (CATI) between January and July 2003. After excluding invalid telephone numbers, persons with insufficient mastery of the German language, and individuals with serious health problems that precluded participation in the interview, we interviewed 2844 of the 4031 persons contacted. The response rate was $71 \%$. For the following analyses, we included only heterosexual participants $(n=2790)$. 


\section{Measures}

\section{Sexual Behavior}

For the previous 12-month period, respondents reported total number of sexual partners and whether they had engaged in different sexual behaviors, such as intercourse with and without condoms, intercourse with casual partners, or with a primary partner with no HIV testing. Sexual risk behavior was defined as sexual intercourse without condom use, with a casual partner, or/and sexual intercourse with a new main partner without condoms or without HIV test for those partners who had ever had intercourse.

For the event-level analysis, respondents provided detailed information on their most recent protected and their most recent unprotected intercourse event with a new partner in the previous 2 -year period. They indicated their cannabis and alcohol use as well as drunkenness before or during the sexual encounter.

\section{Substance Use}

Respondents were asked how often they had consumed cannabis, alcohol, and alcohol to the point of drunkenness in the previous month (never, 1-3 times a month, 1-2 times a week, 3-6 times a week, or daily). As predictors of sexual risk, we included.

\section{Risk Preference and Hedonism as a Personality Trait}

Risk preference and hedonism scale (sumscore of 15 items, Cronbach's alpha $=0.79,0=$ in none of five cases to $5=$ in five of five cases) from the Trier Integrated Personality Inventory developed by Becker (Becker, 2003).

\section{Psychosocial Stress}

Nine items, e.g., stress in school/job or stress with parents, Cronbach's alpha $=0.76,0=$ not at all $10=$ very much .

\section{HIV-related Variables}

Intention to use HIV protection (three items: intention to use condoms with casual partners, intention to use condoms or to take a HIV test with new, sexually experienced main partners, overall intention to use HIV protection, Cronbach's alpha $=0.82$ and self-efficacy concerning condom use, negotiation skills for condom use, general protection against HIV (four items, Cronbach's alpha $=0.51$, the anchors of each scale were $0=$ strongly disagree and $10=$ strongly agree).

\section{Data Analyses}

For the global association study and the mediation models, we defined cannabis users as individuals who consumed cannabis weekly or more than weekly. To compare frequencies of sexual risk behavior among cannabis using and cannabis non-using participants, we used Pearson chi-square tests. Also using Pearson chi-square tests, we tested the hypothesis of a situational effect of cannabis on sexual risk behavior by comparing frequencies of unprotected intercourse and cannabis use prior to the participants' most recent sexual event with a new partner. For the mediation models we followed the procedures of Baron and Kenny (1986). In a first step we computed a logistic regression analysis with sexual risk behavior as the criterion variable and cannabis use as a predictor (path c). In a second step, we computed linear regressions with the mediator as the criterion variable and cannabis use as predictor (path a). In a third step, we used sexual risk behavior as the criterion variable in a logistic regression analysis and cannabis use and the mediator as predictors (path b) and then used a logistic regression analysis with sexual risk behavior as the criterion variable and cannabis use and the mediator as predictors (path $\mathrm{c}^{\prime}$ ). All analyses were controlled for age. To test whether a mediator variable carried the influence of cannabis use to sexual risk behavior, we used the Sobel test. Because gender can be an important moderator for condom use, we also conducted separate analyses for men and women but report separate results only if gender differences appeared. Psychosocial stress correlated only with intention to use HIV protection among young women; we therefore did not compute this mediation model for young men.

\section{RESULTS}

The sample included $52 \%$ young women and $48 \%$ young men. Mean age was 20 years $(\mathrm{SD}=2.46)$, with $38 \%$ of participants aged $16-18$ - 
Table I. Description of Sexual Behavior

\begin{tabular}{lrr}
\hline & $(\%)$ & \multicolumn{1}{c}{$n$} \\
\hline Ever had sexual intercourse & 71.1 & 1985 \\
Intercourse during the previous 12 months & 66.6 & 1859 \\
Casual partners or new main partners & 40.9 & 1141 \\
Risky sexual behavior & 7.2 & 202 \\
Unprotected intercourse with casual & 5.0 & 140 \\
$\quad$ partner $^{a}$ & & \\
Unprotected intercourse with new main $^{\text {partner without HIV testing }}{ }^{a}$ & 2.9 & 81 \\
\hline${ }^{a}$ 19 participants (0.7\%) had unprotected intercourse with both, \\
$\quad$ casual, and new main partners.
\end{tabular}

years-old, $31 \%$ aged $19-21$, and $31 \%$ aged 22 and 24-years-old. Most of the participants were in comprehensive secondary school or at university $(37 \%)$, in professional training (26\%), or employed $(20 \%)$.

Fifty-four percent of the women and $60 \%$ of the men participants had consumed cannabis at least once to date. For the previous 12 -month period, $14 \%$ of the women and $27 \%$ of the men used cannabis at least weekly. Twenty-two percent of the women and $39 \%$ of the men reported drinking to the point of drunkenness at least once in the most recent month.

Table I provides information on frequencies of sexual behavior. There were no differences between men and women. A global association between cannabis use and sexual risk behavior was confirmed. In the most recent year, sexual risk behavior was more frequent among participants who used cannabis at least weekly, 13 vs. $6 \%$ among non-users, $\chi^{2}(1,2737)=36.09, \quad p<0.01$. They had casual partners more often, 30 vs. $15 \%$, $\chi^{2}(1,2730)=62.34, p<0.01$, and more often two or more sexual partners in the previous year, (25 vs. $13 \%), \chi^{2}(1,2745)=47.85, p<0.01$.

\section{Situational Effect of Cannabis on Sexual Risk Behavior}

Fifty-seven percent of the young adults $(n=1587)$ had an intercourse event with a new partner in the previous two years. Most participants had protected intercourse $(n=1456,52 \%) ; 515$ had also unprotected intercourse with a new partner (18\%). Only few of the young adults consumed cannabis in sexual situations. Prior to the most recent protected intercourse event, 84 of 1456 participants $(5.8 \%)$ used cannabis; prior to the most recent unprotected intercourse event, 37 of $515(7.2 \%)$ used cannabis.

Analyzing the most recent intercourse event with a new partner, 336 young adults $(21 \%)$ did not use condoms. Only 88 persons $(5 \%)$ consumed cannabis prior to the intercourse event; there was no significant association between the frequency of cannabis use and the use of condoms in this situation, $\chi^{2}(1,1587)=0.67, p>0.10$. Among participants who consumed cannabis prior to the intercourse event, 64 (75\%) used condoms and 21 persons $(25 \%)$ did not use condoms.

\section{Mediation Models for the Association Between Cannabis Use and Sexual Risk Behavior}

The odds ratio for unprotected sex among women who used cannabis at least once a week compared to non-using women was $2.86, b=1.05$, $\mathrm{SE}=0.24, p<0.01,95 \%$ CI: $1.80-4.52$, and among cannabis-using men 2.18, $b=0.78, \quad \mathrm{SE}=0.21$, $p<0.01,95 \%$ CI: $1.45-3.28$.

Cannabis use was associated with a higher risk preference/hedonism, a lower intention to use HIV protection and a lower HIV self-efficacy (Table II, paths a). Only among women cannabis use was strongly associated with higher psychosocial stress. The strongest predictors for sexual risk behavior were cannabis use, a low intention to use HIV

Table II. Mediation Analyses Based on Separate Logistic Regressions for Explaining Sexual Risk Behavior

\begin{tabular}{|c|c|c|c|c|}
\hline Model & $b$ & SE & $\mathrm{OR} / \mathrm{T}$ & CI $(95 \%)$ \\
\hline \multicolumn{5}{|c|}{ Cannabis use as a predictor for sexual risk behavior } \\
\hline $\mathrm{c}$ & 0.92 & 0.16 & $2.50^{* *}$ & $1.85-3.39$ \\
\hline \multicolumn{5}{|c|}{ Mediator: risk preference/hedonism, Sobel test: $z=4.22^{* *}$} \\
\hline a & 0.44 & 0.05 & $9.55^{* *}$ & $0.35-0.53$ \\
\hline $\mathrm{b}$ & 0.37 & 0.08 & $1.44^{* *}$ & $1.24-1.68$ \\
\hline$c^{\prime}$ & 0.78 & 0.16 & $2.18^{* *}$ & $1.60-3.00$ \\
\hline \multicolumn{5}{|c|}{ Mediator: psychosocial stress (only women), Sobel test: $z=2.37$} \\
\hline a & 0.40 & 0.08 & $5.22^{* *}$ & $0.25-0.55$ \\
\hline $\mathrm{b}$ & 0.29 & 0.11 & $1.34^{* *}$ & $1.09-1.65$ \\
\hline$c^{\prime}$ & 0.98 & 0.24 & $2.67^{* *}$ & $1.67-4.29$ \\
\hline \multicolumn{5}{|c|}{$\begin{array}{l}\text { Mediator: intention to use HIV protection, Sobel test: } \\
z=-4.00^{* *}\end{array}$} \\
\hline $\mathrm{a}$ & -0.30 & 0.07 & $-4.60^{* *}$ & $0.45-0.49$ \\
\hline $\mathrm{b}$ & -0.89 & 0.08 & $0.41^{* *}$ & $0.35-0.49$ \\
\hline$c^{\prime}$ & 0.26 & 0.18 & 1.30 & $0.90-1.86$ \\
\hline \multicolumn{5}{|c|}{ Mediator: HIV self-efficacy, Sobel test: $z=2.65^{* *}$} \\
\hline $\mathrm{a}$ & -0.20 & 0.07 & $-2.94^{* *}$ & $-0.33-0.07$ \\
\hline b & -0.57 & 0.08 & $0.57^{* *}$ & $0.49-0.66$ \\
\hline$c^{\prime}$ & 0.49 & 0.17 & $1.63^{* *}$ & $1.17-2.29$ \\
\hline
\end{tabular}

Path c: cannabis use as predictor for sexual risk behavior Path a: cannabis use as predictor for the mediator variable. Path b: mediator as predictors for sexual risk behavior, controlling for cannabis use.

Path c': effect of cannabis use on sexual risk behavior controlling for the mediator. ${ }^{*} p<0.05 ;{ }^{* *} p<0.01$. 
protection and a low HIV-self-efficacy (Table II, paths b). Risk preference/hedonism and psychosocial stress among women were less important predictors of sexual risk behavior.

The results of the mediation analysis showed that the association between cannabis use and sexual risk behavior was completely mediated by a lower intention to use HIV protection. Thus, the higher frequency of sexual risk behavior among cannabis users can be explained by a lower intention to use HIV prevention of cannabis using young adults compared to non-using persons. Lower HIV-self-efficacy and higher risk preference/hedonism which is more distal from sexual risk behavior were only partial mediators for the association between cannabis use and sexual risk behavior. Only among women the association of cannabis use and sexual risk behavior was partially explained by more psychosocial stress.

\section{DISCUSSION}

Participants who used cannabis engaged in sexual risk behaviors more often in the previous 12-month period than persons who did not use cannabis. Women using cannabis at least weekly were three times more likely to report sexual risk behavior; men were twice as likely to do so. This result agrees with the general finding that individuals using cannabis and other substances engage in sexual risk behaviors more often (see, for example, Bon et al., 2001; Castilla et al., 1999; Poulin and Graham, 2001; Weinhardt and Carey, 2000).

In a combination of event analysis and global association study, we tested whether the association between cannabis use and sexual risk behavior was due to the effect of cannabis in the immediate sexual situation or reflected a more stable risk vulnerability.

The results of the event analyses did not support the hypothesis of a situational influence of cannabis making sexual risk behavior more likely. The assumption of a situational influence of substance use in the model of intention to use HIV protection and HIV preventive behavior (Bengel, 1996), the ARRM (Catania et al., 1990), or in contextual models (Van Campenhoudt, Cohen, Guizzardi, and Hausser, 1997) was not confirmed for cannabis. Cannabis was seldom used prior to the most recent sexual event. Having consumed cannabis, young men and women used condoms just as frequently as when not having consumed cannabis. Condom use or sexual risk behavior seems to be a stable behavior that is independent of cannabis use prior to sexual activity. Weinhardt and Carey (2000) found similar results concerning the effect of alcohol in their review of event-level studies. They concluded that persons who tend to use condoms when they were sober also use them when drinking and that persons who fail to use condoms when drinking probably also fail to use condoms when sober. Thus the more frequent sexual risk behavior among cannabis users seems to be due to a more stable risk vulnerability of these persons that induces both drug use and sexual risk.

To identify person variables that underlie this risk disposition we used mediation analysis. The more frequent sexual risk behavior among cannabis users was completely mediated by a lower intention to use HIV protection, which was also the strongest predictor of sexual risk behavior among cannabis non-using individuals.

Alternatively to the situational effect of cannabis on sexual risk behavior, regular cannabis use might influence-directly or in association with a substance-consuming peer group - the motivational system of a person and in this context also reduce intention to use HIV protection or affect general health behavior. Further research should focus on the mechanisms that lead to a lower intention to use HIV protection among cannabis users.

According to models of multiple risk behavior such as Jessor's Problem Behavior Theory (Jessor, 2001), risk preference and hedonism as a personality trait or psychosocial stress could increase the risk for both cannabis use and sexual risk behavior. In the present study, risk preference/hedonism was a partial mediator for the association between cannabis use and unprotected intercourse. This trait-also in combination with the selection of a peer group - could lead to a more risky lifestyle that includes substance use and sexual risk behavior. Peer norms and partner norms could further increase the preexisting risk preference and hedonism. Other underlying personality traits, such as higher tolerance for deviant behavior or unconventionality and norms of peer groups or parents (Halpern-Felsher, Millstein, and Ellen, 1996), might also cause the more frequent sexual risk among cannabis users and should be tested in further research. Psychosocial stress was a predictor for sexual risk behavior only among young women in our study. Psychosocial stress might render young women more vulnerable to unwanted and unprotected sex than young men, might reduce resources for the safer-sex negotiations that are more important for women. Alternatively, a greater 
number of casual partners, possibly increasing the risk of unprotected sex, might in women be emotion focused coping strategy for psychosocial stress or might ensure social support.

Interventions for preventing sexual risk behavior among young men and women should not regard cannabis use as a situational risk factor for unprotected sex. HIV interventions that include a message on substance use should be careful not to create strong expectancies that substance use leads to sexual risk behavior, for this could promote a self-fulfilling prophecy and have the effect of giving people an excuse for engaging in unprotected sex (Halpern-Felsher et al., 1996). Gender-specific intervention should also focus on the association of psychosocial stress and sexual risk behavior.

Interventions for preventing HIV generally should focus on person variables and enhance the intention to use HIV prevention, self-efficacy, and behavioral skills. Target variables to promote intentions to use HIV protection are perceived behavioral control, attitudes, and subjective norms concerning condom use (Sheeran and Taylor, 1999). Young adults using cannabis at least weekly had decreased intentions to use HIV protection. Thus, target variables for HIV prevention for cannabis users and non-users do not differ. Complementary to this, risk preference and hedonism as an underlying risk disposition for cannabis use and sexual risk behavior might be addressed in interventions for enhancing risk competence and the choosing of exciting activities with less harm potential.

Some limitations of this study should be mentioned. Frequencies of cannabis use and sexual risk behavior were assessed by retrospective questions pertaining to the previous 12-month period. For the event analyses, the period was extended to 24 months, assuring a sufficient number of cases of protected and unprotected sexual intercourse when under the influence of cannabis. The retrospective methodology can lead to a memory bias. The recall of the frequency of past cannabis use could be distorted by the frequency of current cannabis use. Thus, cannabis use prior to sexual intercourse could be over- or under-reported. Data collecting strategies that use diaries containing descriptions of the circumstances of sexual contacts can exclude a memory bias, but as very reactive measures, they may actually influence sexual behavior among young men and women (Weinhardt and Carey, 2000). Our results are consistent with numerous studies which did not find situational effects of alcohol use on sexual risk behavior independent of retrospective or diary based methodology (Weinhardt and Carey, 2000). However, the results of our event-level analysis however should be replicated by other studies. Because of the cross-sectional design of this study, results of the global association study can be interpreted only as correlations and not as causal explanations of sexual risk behavior. To distinguish predictors and consequences of risk behavior, we will conduct a follow-up of the sample in 2005 .

In conclusion, the results of this study found only a general association between substance use and unprotected sexual intercourse that could not be found in event-level analyses. The situational influence of cannabis did not increase sexual risk behavior among young men and women. Cannabis users, however, had decreased intentions to use HIV protection, lower self-efficacy, and a more hedonistic and risky lifestyle, leading to more frequent risky sexual behavior. Thus, the target variables for HIV prevention do not seem to differ for young adults using or not using cannabis.

\section{ACKNOWLEDGMENTS}

This research was funded by the Swiss National Science Foundation (Grant No 3346-65580). The authors would like to thank Sabine Luedi and Eva Schuerch for their contributions during data collection.

\section{REFERENCES}

Ajzen, I. (1991). The theory of planned behavior. Organizational Behavior and Human Decision-Processes, 50(2), 179-211.

Baron, R. M., and Kenny, D. A. (1986). The moderator-mediator variable distinction in social psychological research: Conceptual, strategic, and statistical considerations. Journal of Personality and Social Psychology, 5(6), 1173-1182.

Becker, M. H. (1974). The health belief model and personal health behavior. Thorofare, NJ: Slack.

Becker, P. (2003). TIPI. Trierer Integriertes Persönlichkeitsinventar [TIPI, Trier Integrated Personality Inventory]. Göttingen, Germany: Hogrefe.

Bengel, J. (1996). Risikoverhalten und Schutz vor Aids [Risk behavior and AIDS prevention]. Berlin, Germany: Springer.

Bon, S. R., Hittner, J. B., and Lawandales, J. P. (2001). Normative perceptions in relation to substance use and HIV-risky sexual behaviors of college students. Journal of Psychology, 16(5), 165-178.

Castilla, J., Barrio, G., Belza, M. J., and de la Fuente, L. (1999). Drug and alcohol consumption and sexual risk behavior among young adults: results from a national survey. Drug and Alcohol Dependence, 56, 47-53.

Catania, J. A., Kegeles, S. M., and Coates, T. J. (1990). Towards an understanding of risk behavior: An AIDS risk reduction model (ARRM). Health Education Quarterly, 17(1), 53-72.

Gillmore, M. R., Morrision, D. M., Leigh, B., Hoppe, M. J., Gaylord, J., and Rainley, D. T. (2002). Does "high = high risk"? 
An event-based analysis of the relationship between substance use and unprotected anal sex among gay and bisexual men. AIDS and Behavior, 6(4), 361-370.

Green, B., Kavanagh, D., and Young, R. (2003). Being stoned: A review of self-reported cannabis effects. Drug and Alcohol Review, 22(4), 453-460.

Halpern-Felsher, B. L., Millstein, S. G., and Ellen, J. M. (1996). Relationship of alcohol use and risky sexual behavior: A review and analysis of findings. Journal of Adolescent Health, 19(5), 331-336.

Hawkins, J. D., Catalano, R. F., and Miller, J. Y. (1992). Risk and protective factors for alcohol and other drug problems in adolescence and early adulthood: Implications for substance abuse prevention. Psychological Bulletin, 112(1), 64-105.

Jessor, R. (2001). Problem-behavior-theory. In J. Raithel (Ed.), Risikoverhaltensweisen Jugendlicher [Risk behaviors of young persons] (pp. 61-78) . Opladen: Leske + Budrich.

Jessor, R., and Jessor, S. L. (1977). Problem behavior and psychosocial development: A longitudinal study of youth. New York: Academic Press.

Kelleher, L. M., Stough, C., Sergejew, A. A., and Rolfe, T. (2004). The effects of cannabis on information-processing speed. $A d$ dictive Behaviors, 29(6), 1213-1219.

Kleiber, D., and Soellner, R. (1998). Cannabiskonsum. Entwicklungstendenzen, Konsummuster und Risiken [Cannabis usage, consumption patterns, and risks]. München, Germany: Juventa.

Leigh, B. (1999). The risks of drinking among young adults. Peril, chance, adventure: concepts of risk, alcohol use and risky behavior in young adults. Addiction, 94(3), 371383.

Poulin, C., and Graham, L. (2001). The association between substance use, unplanned sexual intercourse and other sexual behaviors among adolescent students. Addiction, 96, 607621.

Rogers, R. W. (1983). Cognitive and physiological processes in fear appeals and attitude change: A revised theory of protection motivation. In J. T. Capioppo, and D. R. Petty (Eds.), Social psychophysiology (pp. 153-176). New York: Guilford.

Sheeran, P., Abraham, C. S., and Orbell, S. (1999). Psychosocial correlates of heterosexual condom use: A meta-analysis. Psychological Bulletin, 125, 90-131.

Sheeran, P., and Taylor, S. (1999). Predicting intentions to use condoms: A meta-analysis and comparison of the theories of reasoned action and planned behavior. Journal of Applied Social Psychology, 29(8), 1624-1675.

Stall, R., McKusick, L., Wiley, J., Coates, T. J., and Ostrow, D. G. (1986). Alcohol and drug use during sexual activity and compliance with safe sex guidelines for AIDS: The AIDS behavioral research project. Health Education Quarterly, 13(4), 359-371.

Staton, M., Leukefeld, C., Logan, T. K., Zimmerman, R., Lynam, D., Milich, R., et al. (1999). Gender differences in substance use and initiation of sexual activity. Population Research and Policy Review, 18, 89-100.

Van Campenhoudt, L., Cohen, M., Guizzardi, G., and Hausser, D. (1997). Sexual interaction and HIV risk. New conceptual perspectives in European research. London: Taylor and Francis.

Weinhardt, L. S., and Carey, M. P. (2000). Does alcohol lead to sexual risk behavior? Findings from event-level research. Annual Review of Sex Research, 11, 125-157.

Wingood, G. M., and DiClemente, R. J. (1998). The influence of psychosocial factors, alcohol, drug use on African-American women's high-risk sexual behavior. American Journal of Preventive Medicine, 15(1), 54-59. 